\title{
ESTUDOS PRELIMINARES DE MATERIAIS TERMOELÉTRICOS PARA OBTENÇÃO DE ENERGIA ELÉTRICA A PARTIR DO CALOR RESIDUAL
}

\author{
Renato Américo ${ }^{\prime}$ \\ Lírio Schaeffer ${ }^{2}$ \\ Edemar Ouriques \\ Gerson Costa
}

\section{Resumo}

O presente artigo consiste em um estudo preliminar dos efeitos de geradores termoelétricos baseados no princípio de aplicação do efeito Seebeck para a geração de energia elétrica. Para tanto, foi realizado um levantamento bibliográfico acerca do assunto, buscando avaliar as características principais e as possibilidades de aplicações. De um modo conciso, são apresentados alguns resultados preliminares quanto a produção de amostras da liga bismuto-antimônio-zinco, produzidas através de pós obtidos pelo processo da Metalurgia do pó. As mesmas foram desenvolvidas com o intuito de serem objetos de estudos na sequência da pesquisa, afim de conhecer as características e propriedades termoelétricas desses materiais após serem compactados. Os primeiros resultados foram obtidos através da medição da tensão elétrica de cada composição analisada.

Palavras-chave: Geradores termoelétricos; Efeito seebeck; Energia; Reaproveitamento de energia.

\section{PRELIMINARY STUDIES OF THERMOELECTRIC MATERIALS FOR THE OBTAINMENT OF ELECTRICAL ENERGY FROM RESIDUAL HEAT}

\begin{abstract}
\section{INTRODUÇÃO}

Atualmente há diversos tipos de fontes de energia em uso no mundo, como exemplo, energia eólica, nuclear, hidráulica, biomassa, solar, entre outras, sendo que algumas são limitadas e/ou esgotáveis, como energia fóssil (derivados de petróleo e carvão mineral). Isto nos atenta na busca de fontes alternativas de energia, concentrando os esforços em energias renováveis. Dentre os mais variados tipos energias renováveis, grande destaque se dá à geração de energia elétrica.
\end{abstract}

The present paper consists of a preliminary study of the effects of thermoelectric generators based on the principle of application of the Seebeck effect for the generation of electric energy. To do so, a bibliographic survey about the subject was carried out, aiming to evaluate the main characteristics and the possibilities of applications. In a concise way, some preliminary results are presented regarding the production of samples of the bismuth-antimony-zinc alloy, produced through powders obtained by the powder metallurgy process. They were developed with the intention of being objects of studies in the sequence of the research, in order to know the characteristics and thermoelectric properties of these materials after being compacted. The first results were obtained by measuring the voltage of each analyzed composition. Keywords: Thermoelectric generators; Seebeck effect; Energy; Energy reuse.

Nesse sentido, buscando contribuir para melhorias nesse contexto, é objeto desse estudo a conversão de calor - com destaque para o calor residual ou calor perdido - em energia elétrica, através do uso de geradores termoelétricos (TEG - Thermoeletric Generators).

De modo sucinto, os TEG's são dispositivos construídos com materiais semicondutores e metais, afim de garantir uma diferença de temperatura entre as faces (lado quente e lado frio), resultando em uma diferença de potencial

'Escola de Engenharia, Universidade Federal do Rio Grande do Sul - UFRGS, Porto Alegre, RS, Brasil. E-mail: renato.americo30@hotmail.com 2Escola de Engenharia, Universidade Federal do Rio Grande do Sul - UFRGS, Porto Alegre, RS, Brasil. E-mail: schaefer@ufrgs.br 
(corrente elétrica). Um simples termopar é fundamentado neste princípio, onde para uma determinada temperatura, os dois materiais do sensor geram uma diferença de potencial que é lida pelo aparelho de medição. Esse efeito pode ser explicado pelo fenômeno conhecido como "Efeito Seebeck".

Desta forma, o estudo visa contribuir para a diminuição do desperdício de calor gerado por máquinas e equipamentos, contribuindo também com a redução no consumo de energias fósseis e na geração de gás carbônico, tão prejudicial para a vida humana.

\section{HISTÓRICO E CARACTERÍSTICAS}

\section{I O efeito Seebeck}

Ao observar efeitos eletromagnéticos em circuitos de bismuto/cobre e bismuto/antimônio, Seebeck descobriu a existência de correntes termoelétricas, quando junções de dois metais dessemelhantes são expostos em circuito fechado, a temperaturas diferentes. Nesse caso, sob a aplicação de uma diferença de temperatura, ocorre o movimento dos elétrons livres da extremidade quente para a extremidade fria, gerando uma tensão elétrica, proporcional à diferença de temperatura [I]. O esquema representado na Figura I demonstra esse efeito.

Materiais diferentes apresentam comportamentos distintos em relação ao fenômeno. Assim, sua quantificação é denominada de Coeficiente de Seebeck e pode ser calculada pela Equação I:

$$
\alpha=\frac{\Delta V}{T_{1}-T_{2}}
$$

Onde:

- $\alpha$ é o coeficiente de Seebeck (V/K)

- $\Delta$ V é a tensão elétrica gerada pela diferença de temperatura $(V)$

- $T_{1}$ e $T_{2}$ são as temperaturas nas quais o material está submetido $(\mathrm{K})$

\subsection{Características Importantes dos Geradores Termoelétricos}

Análogo ao exposto, conhecidos como módulos termoelétricos, os TEG's, são constituídos por materiais semicondutores e metais que realizam a tarefa de gerar energia elétrica a partir de energia térmica, conforme ilustra a Figura 2, podendo ser do tipo $\mathrm{N}$ ou do tipo $\mathrm{P}$, se caracterizam por gerarem correntes elétricas iguais, porém, em sentido contrário. Construídos com uma cerâmica isolante, ausente de partes móveis, esses dispositivos podem ser usados em qualquer fonte que libere calor, ainda que esse calor seja excedente de sua operação normal, como no caso de motores e canos de descarga de automóveis, tubulações de vapor, fornos industriais, etc. [2,3].

Alguns aspectos importantes dos Módulos de Geradores Termoelétricos podem ser destacados como [4]:
- Confiáveis e silenciosos, não possuindo partes móveis;

- Requerem pouca manutenção;

- Tamanhos e pesos reduzidos;

- Possuem longo tempo de vida;

- São ecologicamente corretos.

Os materiais termoelétricos utilizados atualmente têm eficiência típica - sua capacidade de converter calor em eletricidade - entre 5 e $6 \%$. Uma nova geração de materiais está levando essa eficiência para a faixa entre II e I4\%" [I,5].

Para que o desempenho dos materiais termoelétricos seja avaliado, a dimensão utilizada é chamada de "Figura de Mérito (Z ou ZT)", que tem seu resultado influenciado por três características do material termoelétrico: elevado coeficiente de Seebeck $(\alpha)$, alta condutividade elétrica $(\sigma)$ e baixa condutividade térmica $(k)$. Seu resultado também difere de acordo com o material e a temperatura.

Assim, a Figura de Mérito (ZT) pode ser calculada pela Equação 2 [6]:

$$
Z T=\frac{\sigma \cdot \alpha^{2}}{k}
$$

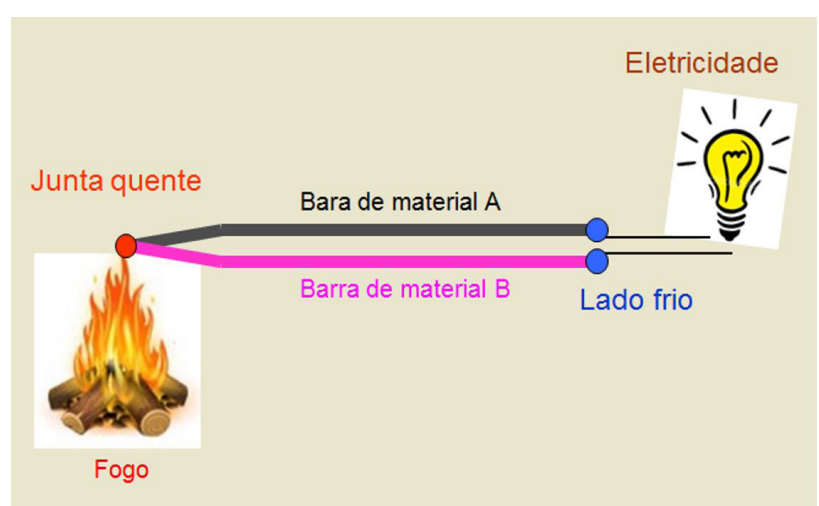

Figura I. Forma simplificada demonstrando o Efeito Seebeck.

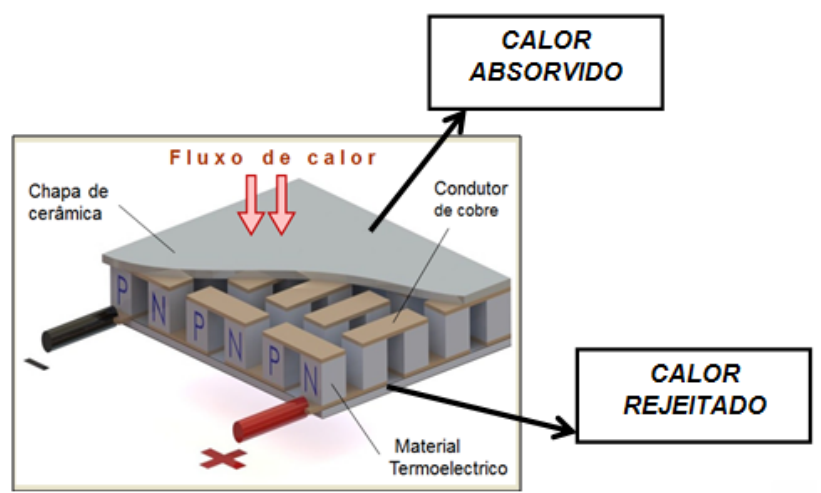

Figura 2. Gerador Termoelétrico (ou Módulo Termoelétrico) [7]. 
Onde:

- $\sigma$ é Condutividade Elétrica $(\mathrm{S} / \mathrm{m})$

- $\alpha$ é o Coeficiente de Seebeck (V/K)

- $k$ é a Condutividade Térmica $(\mathrm{W} /(\mathrm{mK}))$

Um material para ser eficiente do ponto de vista termoelétrico, terá que possuir elevado coeficiente de Seebeck e condutividade térmica e resistividade elétrica baixa".

Os melhores valores de ZT são obtidos em materiais semicondutores fortemente dopados, tais como as ligas $\mathrm{Bi}_{2} \mathrm{Te}_{3}$, $\mathrm{PbTe}$ e SiGe. No caso da utilização à temperatura ambiente, de 200 a 350K, os compostos envolvendo Telúrio (Te), Bismuto (Bi), Antimônio ( $\mathrm{Sb}$ ) e Selênio (Se) apresentam maior figura de mérito, conforme Figura 3, justificando assim, seu uso na maioria dos dispositivos comerciais [5].

\section{PROCEDIMENTOS EXPERIMENTAIS}

\section{I Ciclo Produtivo das Amostras}

A Figura 4 ilustra de uma forma esquematizada o ciclo produtivo de amostras em laboratório (Laboratório de Transformação Mecânica /UFRGS), respeitando cada etapa e composição dos materiais para fabricação da amostra final. O procedimento de fabricação de um TEG compõe-se de:

$\checkmark$ Componentes “pós”: (Bismuto, Antimônio, Zinco, Carbetos de Silício, Cobre e Telúrio);

$\checkmark$ Moagem para obter mesma granulometria do pó;

$\checkmark$ Mistura ou homogeneização dos materiais;

$\checkmark$ Matrizes (Ferramentas para compactação);

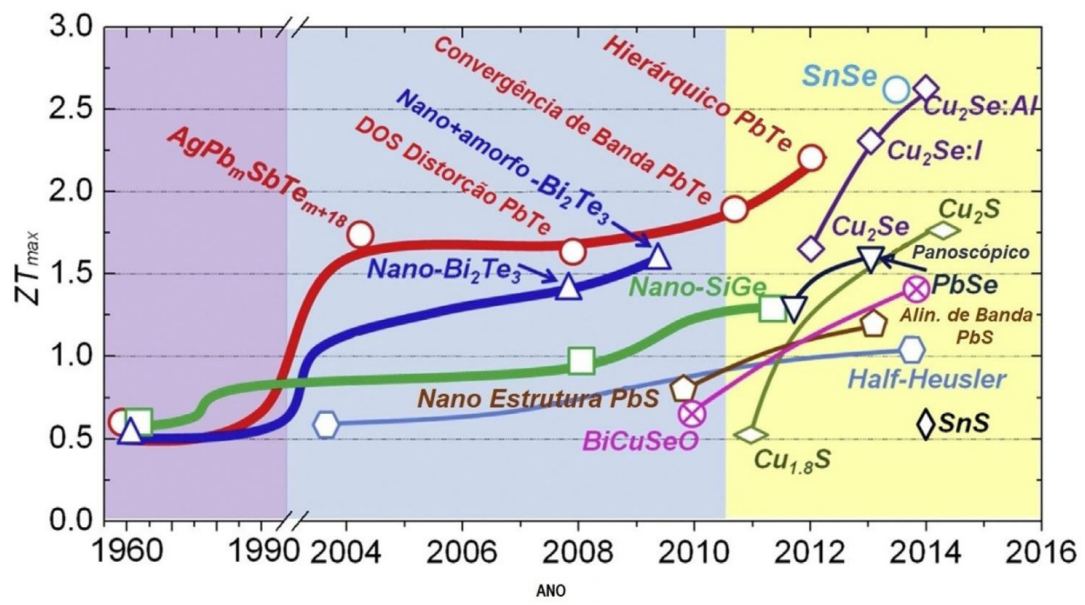

Figura 3. Materiais termoelétricos com a figura de mérito (ZT) ao passar dos anos [8].

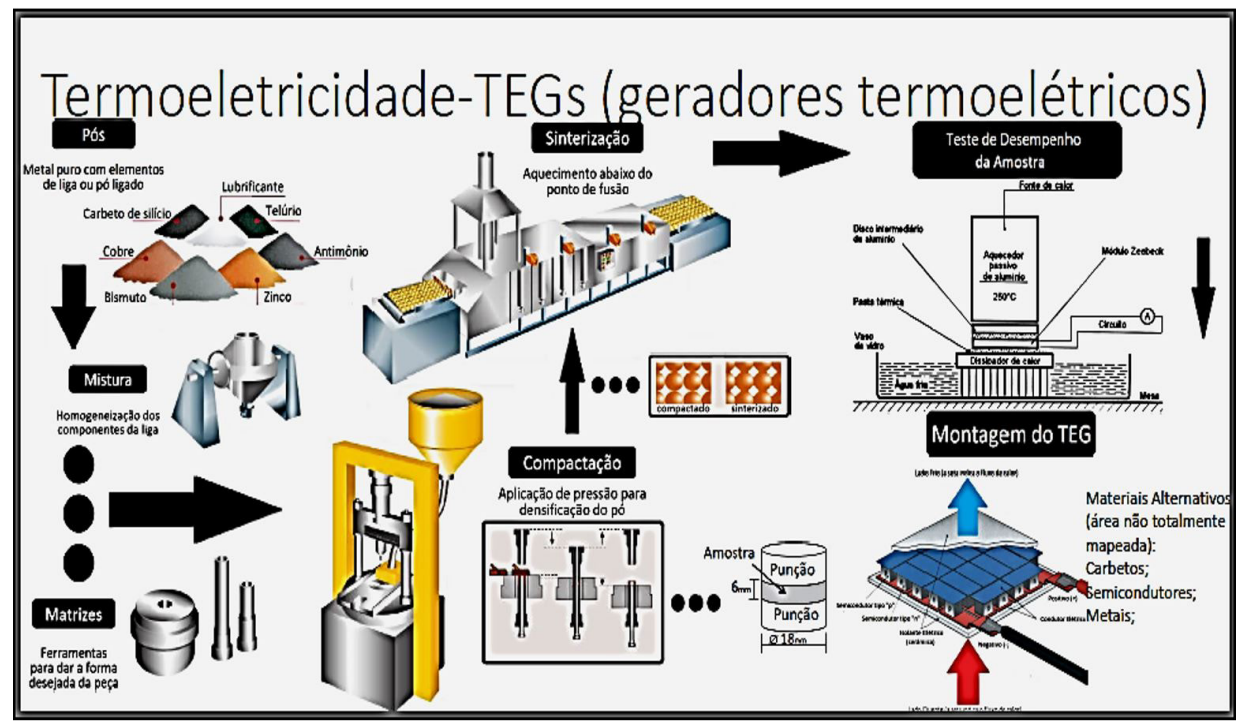

Figura 4. Ciclo produtivo em laboratório. 
$\checkmark$ Prensa hidráulica para 0 uso das ferramentas de compactação;

$\checkmark$ Sinterização (aquecimento em fornos);

$\checkmark$ Teste de desempenho da amostra em bancada;

$\checkmark$ Montagem final do Termoeletrogerador (TEG).

\subsection{Preparação das Amostras}

Novas amostras são obtidas a partir da metalurgia do pó, porém deve-se definir uma composição adequada. Através de uma balança de precisão, faz-se a pesagem com o percentual de cada componente desejado. É importante ter uma uniformidade granulométrica de cada componente, obtida através de moagem.

Após a formação da composição, mistura-se tudo em um misturador helicoidal por 2 minutos, com a adição de lubrificante a mistura (aproximadamente $1 \%$ da massa total da amostra) para facilitar a compactação. Então o pó é compactado em uma matriz circular de diâmetro $18 \mathrm{~mm}$

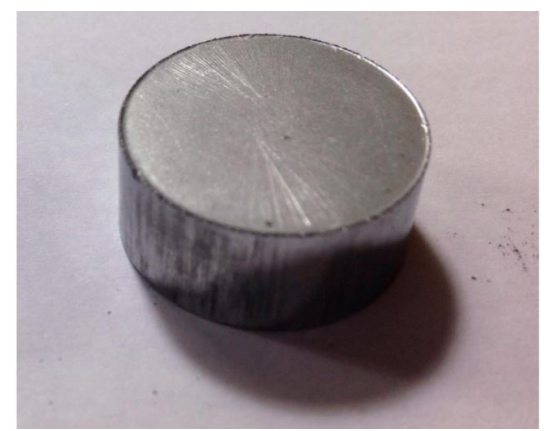

Figura 5. Amostra obtida por compactação do pó da liga BiSbZn. ou $13 \mathrm{~mm}$, conforme desejado. A pressão de compactação utilizada como padrão foi de aproximadamente $730 \mathrm{MPa}$.

Com a amostra já compactada, o lubrificante é removido através do processo de eliminação do lubrificante, em um forno com uma taxa de aquecimento de aproximadamente $5^{\circ} \mathrm{C} / \mathrm{min}$, a uma temperatura de $80^{\circ} \mathrm{C}$. A amostra foi mantida nessa temperatura por pelo menos I hora para que todo 0 lubrificante fosse removido (Figura 5).

\subsection{Bancada de Testes}

O objetivo inicial da bancada de teste nesta pesquisa, foi analisar a tensão gerada por cada amostra, junto com as temperaturas do lado quente e do lado frio. $\mathrm{O}$ aquecedor construído no laboratório pode chegar a temperatura de $400^{\circ} \mathrm{C}$ (Figura 6).

\subsection{Medição da tensão e corrente}

Com a amostra pronta, o aquecedor, que funciona por indução, fabricado pela equipe de pesquisa, do laboratório de termoeletricidade da UFRGS, tem uma potência de $464,215 \mathrm{~J} / \mathrm{s}$. Quando o aquecedor atinge uma temperatura superior a $420^{\circ} \mathrm{C}$, ele é colocado sobre a amostra e o experimento é iniciado.

A partir do momento em que o aquecedor entra em contato com a amostra, a tensão gerada começa a aumentar gradativamente até estabilizar, com aplicação de multímetros, então a potência máxima é medida. Para medir a potência, o circuito é fechado com um potenciômetro de I $\mathrm{k} \Omega$ e dois multímetros ligados em série. Além disso, a tensão entre a entrada e a saída do potenciômetro é medida, assim quando a resistividade é variada, a tensão e a corrente são medidas, conforme ilustra a Figura 7 e 8.

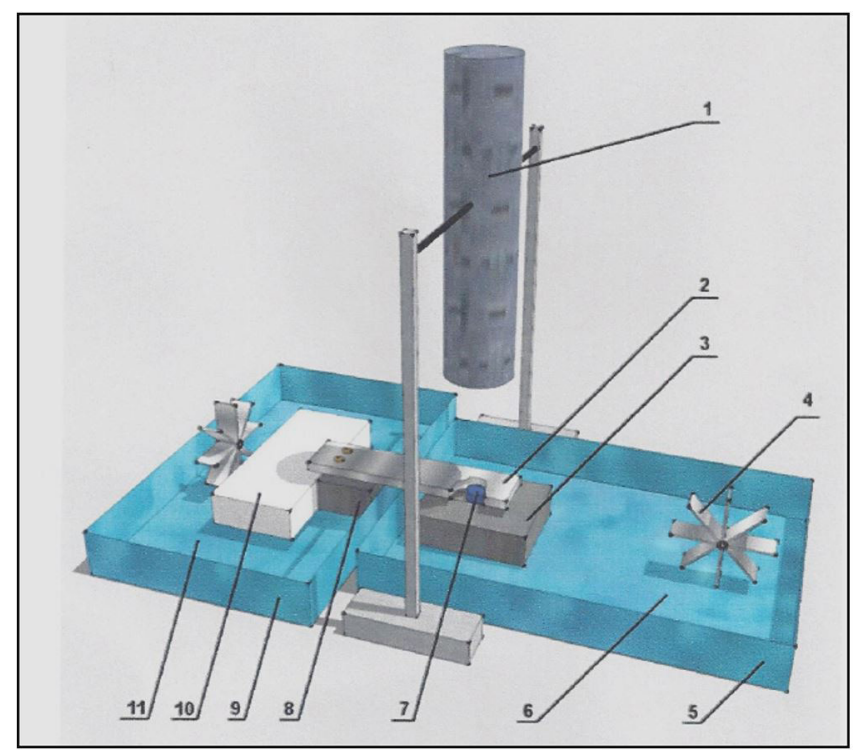

Figura 6. Vista geral da montagem experimental. I - Aquecedor de resistência elétrica; 2 - Braço de aço; 3 - Dissipador de calor I; 4 - Bomba de água "agitador"; 5 - Vaso de água fria I; 6 - Água fria; 7 - Amostra testada; 8 - Dissipador de calor 2; 9 - Vazo de água fria 2; 10 - Apoio flutuante de braço de ferro "tipo boia"; 1 I - Água fria. 


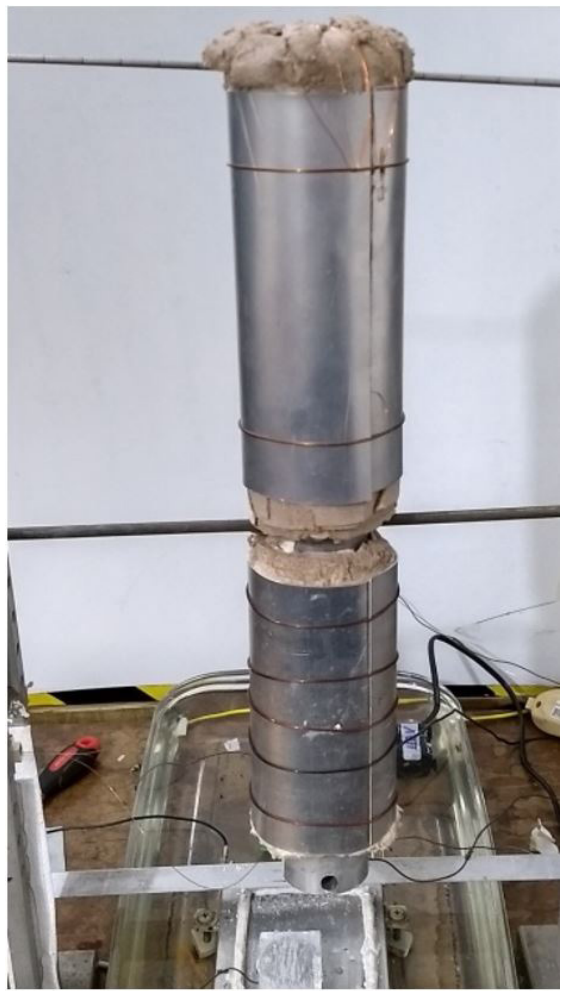

Figura 7. Bancada inicial para teste.
Os dados de temperatura são obtidos através de termopares tipo K, ligados em um aquisitor de dados “Field Logger", fabricado pela NOVUS, Figura 9.

Nesse aparelho são obtidos dados da temperatura do aquecedor, da superfície superior da amostra, do dissipador que fica abaixo da amostra, do ambiente (aproximadamente $23^{\circ} \mathrm{C}$ ) e das águas dos dois recipientes. No software do dispositivo, foi criado um canal virtual chamado "Gradiente" onde ele subtrai a temperatura do dissipador da temperatura superior da amostra. Os multímetros possuem precisão de $\mathrm{I} 00 \mu \mathrm{V}$ e I $\mu \mathrm{A}$, e os termopares tem uma faixa de erro de $\mathrm{I}^{\circ} \mathrm{C}$.

\section{RESULTADOS E DISCUSSÕES}

Para este trabalho inicial foram definidas 16 composições da liga bismuto-antimônio e zinco (BiSbZn), sendo os materiais carbetos de silício e telúrio, serão aplicados em amostras em uma segunda etapa da pesquisa.

As 16 amostras foram utilizadas com um percentual diferente de elementos contidos na liga, previamente escolhidos. A composição variou de 3 a $84 \%$ de bismuto, 16 a $84 \%$ de antimônio e $0 \%$ a $35 \%$ de zinco, com massas variando de 6,22 à 19,8g (conforme Tabela I).

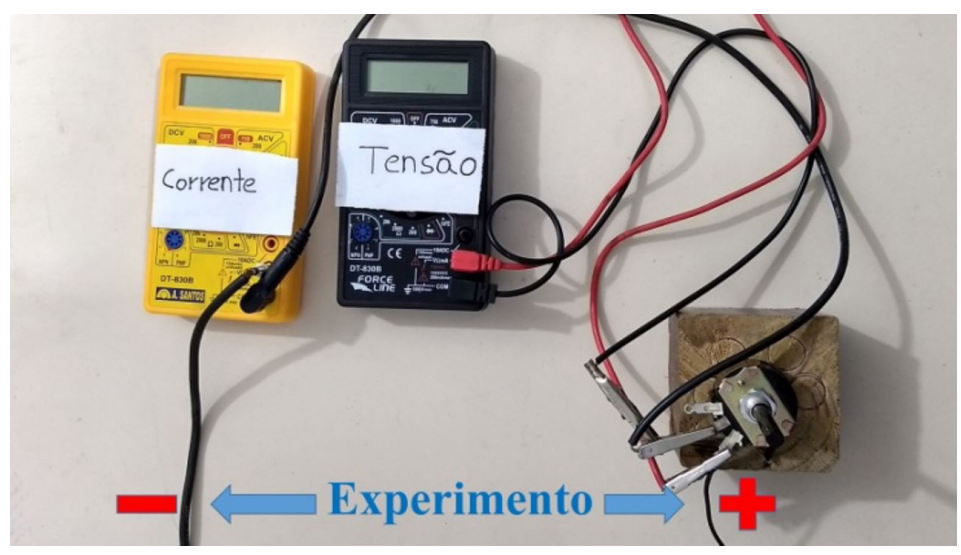

Figura 8. Aparelhos de medição de tensão e corrente.

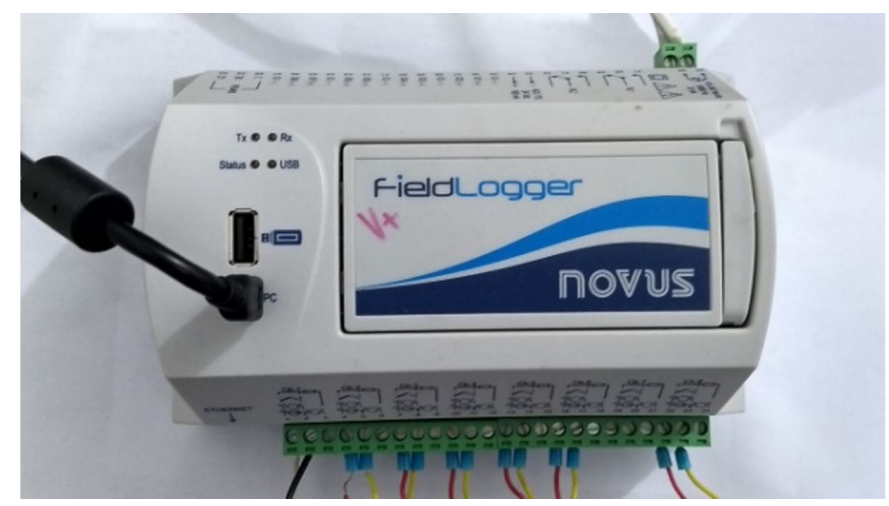

Figura 9. Aquisitor de dados. 
Tabela I. Quantidades experimentais para amostras BiSbZn

\begin{tabular}{|c|c|c|c|c|c|c|c|c|c|}
\hline Amostra & Bi (\%) & Sb (\%) & Zn (\%) & $\begin{array}{c}\text { Altura } \\
(\mathrm{mm})\end{array}$ & $\begin{array}{c}\begin{array}{c}\text { Diâmetro } \\
(\mathrm{mm})\end{array} \\
\end{array}$ & $\begin{array}{c}\text { Área } \\
\left(\mathrm{mm}^{2}\right)\end{array}$ & Massa (g) & $\begin{array}{c}\text { Compactação } \\
(\mathrm{MPa})\end{array}$ & $\begin{array}{c}\text { Tensão } \\
(\mathrm{mV})\end{array}$ \\
\hline Al & 5 & 70 & 25 & 12,50 & 18,27 & 262,16 & 19,80 & 732 & 11,8 \\
\hline A2 & 10 & 60 & 30 & 3,75 & 18,12 & 257,87 & 6,22 & 732 & 11,6 \\
\hline A3 & 15 & 50 & 35 & 4,31 & 18,13 & 258,16 & 7,37 & 732 & 10,3 \\
\hline A4 & 16 & 84 & 0 & 5,56 & 18,12 & 257,87 & 9,82 & 732 & 7,0 \\
\hline A5 & 84 & 16 & 0 & 5,00 & 18,7 & 274,65 & 9,15 & 732 & $-7,0$ \\
\hline Al.I & 5 & 70 & 25 & 5,79 & 18,16 & 259,01 & 9,80 & 732 & 15,4 \\
\hline A2.1 & 10 & 60 & 30 & 5,77 & 19,09 & 286,22 & 8,50 & 732 & 11,6 \\
\hline A6 & 3 & 72 & 25 & 6,16 & $18, \mid$ & 257,30 & 9,83 & 732 & 15,5 \\
\hline A7 & 7 & 68 & 25 & 6,38 & 18,11 & 257,59 & 9,80 & 732 & 14,3 \\
\hline A8 & 3 & 72 & 25 & $|3,4|$ & 18,33 & 263,90 & 19,95 & 732 & 14,4 \\
\hline A9 & 4 & 71 & 25 & 7,11 & 18,14 & 258,40 & 9,97 & 732 & 12,7 \\
\hline A9.1 & 4 & 71 & 25 & 6,19 & 18,15 & 258,70 & 9,92 & 732 & 14,7 \\
\hline Alo & 2 & 73 & 25 & 7,20 & 18,12 & 257,90 & 10,06 & 732 & 12,2 \\
\hline AlI & 3,46 & 71,65 & 24,88 & 6,69 & 20,34 & 162,50 & 5,16 & 732 & 25,7 \\
\hline$A \mid 2$ & I & 58 & 41 & 6,28 & 18,17 & 259,30 & 9,75 & 732 & 34,3 \\
\hline Al3 & I & 58 & 41 & 6,38 & 18,14 & 258,40 & 9,89 & 582 & 20,2 \\
\hline
\end{tabular}

A amostra AI2, foi a que apresentou o melhor resultado em relação a tensão gerada de $34,3 \mathrm{mV}$, sendo a mesma fabricada com os mesmos métodos, alterando somente sua composição, com os percentuais do $\mathrm{Bi}$, $\mathrm{Sb}$ e $\mathrm{Zn}$ e com alteração da altura e massa.

\section{CONCLUSÕES}

As amostras que apresentaram melhores resultados em relação à tensão gerada, são as que possuem um percentual baixo de Bismuto. Esse resultado é excelente, uma vez que o Bismuto é o elemento de mais alto custo.

Os resultados acima não foram analisados a granulometria das amostras, pois influenciam na compactação e espaços intersticiais, podendo aumentar a condutividade térmica, e não foram avaliadas as geometrias, trabalhando apenas com a matriz de $18 \mathrm{~mm}$.

Os testes analisados inicialmente, mensurando apenas tensão e corrente, não analisados as porcentagem efetiva de cada componente na amostra por FRX e microestrutura em DRX.
A composição da amostra Al 2 evidenciou a maior tensão gerada, apontou um ponto positivo para a pesquisa. A busca por melhores desempenhos com a liga BiSbZn na geração de energia elétrica é um grande desafio da equipe. Os objetivos futuros seguem o caminho da busca de resultados mais significativos com uma melhor composição, analisando altura e massa das amostras, tipos de geometria, trabalhando com materiais de baixo custo de produção, aplicando em amostras o carbeto de silício $(\mathrm{SiC})$ e o telúrio $(\mathrm{Te})$.

A redução das partículas dos materiais, também será um grande desafio da equipe, utilizar os processos de redução com moinhos de bolas, para redução em nano partículas, para melhorar a redução da condutividade térmica das amostras compactadas, reduzindo os espaços intersticiais.

Sendo um próximo passo obter um procedimento de dopagem das amostras tipo $\mathrm{P}$ e amostras tipo $\mathrm{N}$, intensificar os estudos nos processos de sinterização, homogeneização e granulometria dos materiais, para verificação da energia gerada em watts, calcular a figura de mérito das amostras selecionadas, avaliando o seu rendimento em relação ao já existente no mercado.

\section{REFERÊNCIAS}

I Priya S, Inman JD. Energy harvesting technologies. USA: Springer Science+Business Media LLC.; 2009

2 Farias SRA. Protótipo de um microgerador termoelétrico de estado sólido: cogeração a gás (dissertação). Natal: Universidade Federal do Rio Grande do Norte; 2009

3 Polozine A, Schaeffer L. Materiais sinterizados para geração de energia elétrica. In: Anais do $33^{\circ}$ SENAFOR: " $10^{\circ}$ Encontro de Metalurgia do Pó/4a COMEP - Conferência Internacional de Metalurgia do Pó - Brasil - RS”; 2013 outubro 9-II; Porto Alegre, Brasil. Porto Alegre: UFRGS - LdTM; 2013.

4 Véras JCC. Análise experimental dos efeitos de ciclostérmicos em geradores termoelétricos [dissertação]. João Pessoa: Universidade Federal do Paraíba; 2014. 
Américo et al.

5 Almeida CHA. Caracterização de célula termoelétrica para geração de energia elétrica [dissertação]. João Pessoa: Universidade Federal da Paraíba; 2015.

6 Chen Z-G, Han G, Yang L, Cheng L, Zou J. Nanostructured thermoelectric materials: current research and future challenge. Austrália: Materials International; 2012. p. 535-549.

7 Min G. Thermoeletrecy Energy Harvesting. Artech House. 20I0;4I3-414:325-336.

8 Zhang X, Zhao L-D. Energy conversion between heat and electricity. Journal of Materiomics. 2015; 1:92-105.

Recebido em: 13 Set. 2017

Aceito em: 06 Mar. 2018 